\title{
Delayed dislocation of a sutureless aortic bioprosthesis: the first case
}

\author{
Department of Adult Cardiac Surgery, G. Pasquinucci Heart Hospital, Massa, Italy \\ * Corresponding author. Tel: +39-0585-493604; fax: +39-0585-493604; e-mail: gioconci@libero.it (G. Concistrè).
}

Giovanni Concistrè*, Antonio Miceli, Francesca Chiaramonti and Mattia Glauber

Received 5 July 2011; received in revised form 2 September 2011; accepted 7 September 2011

\begin{abstract}
Sutureless aortic bioprosthesis implantation is an alternative technique in high-risk patients undergoing aortic valve replacement with a possible reduction in the extracorporeal circuit time and reliable haemodynamic features. A 3F Enable (ATS Medical-Medtronic, Inc., Minneapolis, MN, USA) has shown very good results. We report the first upward displacement of $3 \mathrm{~F}$ Enable three months post implantation.
\end{abstract}

Keywords: Aorta • Aortic valve replacement $\cdot$ Bioprosthesis

\section{INTRODUCTION}

Aortic valve replacement (AVR) with mechanical or biological stented valves is the standard treatment for patients with severe, symptomatic aortic stenosis [1]. However, high-risk patients undergoing AVR may require alternative treatment options to reduce postoperative mortality and morbidity. Sutureless aortic bioprosthesis implantation is a less-invasive technique, which may shorten the cardiopulmonary bypass (CPB) time and show an excellent haemodynamic performance. Results are encouraging and this technique continues evolving. The rate of perivalvular leakage remains an important issue with this technology [2], but in literature there are no described cases of the sutureless dislocation requiring reintervention. We report the first case of a late upward displacement of a $3 \mathrm{~F}$ Enable three months post implantation.

\section{CASE REPORT}

A 78-year old woman was referred to our hospital for correction of symptomatic severe aortic stenosis and left anterior descending (LAD) coronary artery stenosis. The body surface area was $1.37 \mathrm{~m}^{2}$ and logistic EuroSCORE was $10.3 \%$. Chest radiography showed moderate cardiomegaly. The patient was on sinus rhythm. Transthoracic echocardiography showed a tricuspid aortic valve with evidence of severe stenosis (orifice area: 0.7 $\mathrm{cm}^{2}$ ). The mean aortic transvalvular pressure gradient was 79 $\mathrm{mmHg}$ and the aortic annulus diameter was $23 \mathrm{~mm}$. Left ventricular (LV) function was preserved with the sign of LV hypertrophy. Angiography demonstrated severe aortic valve stenosis with evidence of critical LAD stenosis.

After median sternotomy, the CPB was instituted with aorto-atrial cannulation. The heart was arrested with retrograde normothermic blood cardioplegia and LAD was revascularized with left mammary artery. The transverse aortotomy was performed $\sim 3 \mathrm{~cm}$ above the sinotubular ridge and the aortic valve was inspected. The valve was tricuspid and extremely calcified. The native aortic valve was excised, the annulus was decalcified and the valve was replaced with a $3 F$ Enable (ATS Medical-Medtronic, Inc., Minneapolis, MN, USA) 23-mm sutureless bioprosthesis. The rising and folding processes of the prosthesis and the implantation technique have been described by Martens et al. [2]. The aortotomy was closed with a continuous 4-0 prolene suture and the patient was weaned from the CPB uneventfully. The CPB time and aortic cross-clamp (ACC) time were 103 and $70 \mathrm{~min}$, respectively. Intraoperative transoesophageal echocardiography showed a good position and normal valve function with mild perivalvular leakage.

Postoperative course was uneventful. At discharge, the echocardiogram showed a normal aortic bioprosthesis function with the persistence of mild perivalvular leakage.

Three months after the operation, the patient had congestive heart failure associated with haemolytic anaemia. Echocardiography showed severe aortic perivalvular regurgitation with the sutureless bioprosthesis dislocation at the level of left sinus of Valsalva (Fig. 1). The patient was transfused and, after haemodynamic stabilization, surgery was re-planned.

After median sternotomy and lysis of tenacious adhesions, the CPB was instituted, the heart was arrested and the transverse aortotomy was performed as described previously. At the follow-up, a dislocation into the outflow tract was confirmed with the displacement at the level of left sinus of Valsalva. The bioprosthesis was intact and showed no degeneration. The intimal wall of the aortic root did not show injury. The 3F Enable was easily mobilized by irrigation with chilled sterile physiological saline solution to keep the Nitinol frame malleable, and then correctly repositioned and expanded with warmer saline. We fixed the bioprosthesis in the aortic annulus with four pledgeted stitches. The CPB and ACC time were 54 and 28 min, respectively. Intraoperative transoesophageal echocardiography showed a good position and normal function of the bioprosthesis 


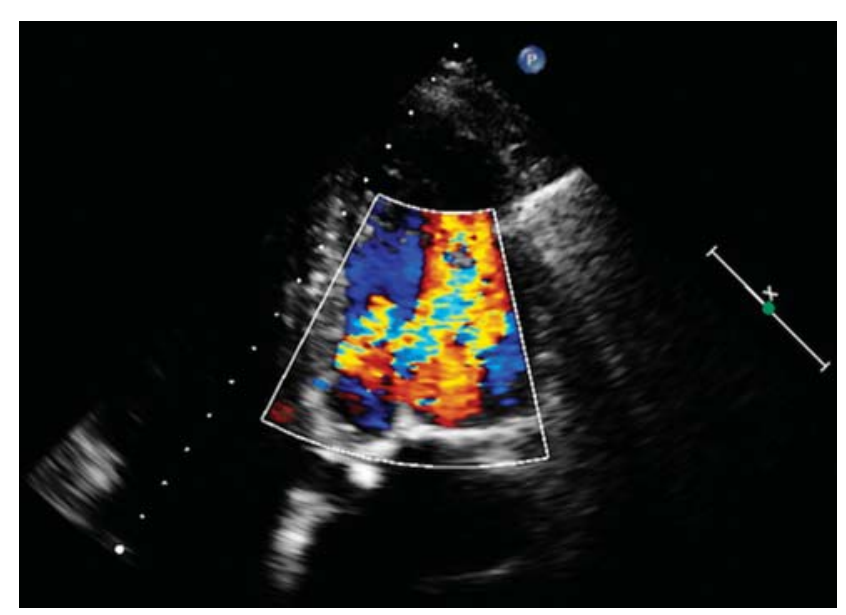

Figure 1: Severe aortic perivalvular regurgitation with the sutureless bioprosthesis dislocation.

without perivalvular leakage. Postoperative course was uneventful.

\section{COMMENT}

To the best of our knowledge, this is the first case reported in literature of a late $3 \mathrm{~F}$ Enable sutureless dislocation. We believe that the dislocation was not linked to the size of the bioprosthesis, but probably due to the deep decalcification determining an anatomic inexact circular aortic annulus and consequently an incorrect implantation. Realizing that the bioprosthesis was intact and the leaflets were not degenerated, we decided to re-implant the same valve. Specifically, the bioprosthesis was irrigated with chilled saline solution to make the Nitinol frame malleable and repositioned at the level of the aortic annulus. Subsequently, it was expanded with warmer saline and the bioprosthesis was fixed into the aortic annulus with some pledgeted stitches, to reduce the risk of re-migration.

The 3F Enable sutureless aortic valve bioprosthesis represents an innovative approach for surgical AVR in high-risk patients [3-5]. As the transcatheter valve technology has developed rapidly in the past few years, the devices for surgical AVR have also improved. Advantages of the $3 \mathrm{~F}$ Enable prosthesis are the large valve orifice area, comparable with stentless valves (the nitinol stent is only $1 \mathrm{~mm}$ thick), accurate decalcification of the native annulus as well as accurate positioning of the prosthesis and potential fast implantation with respect to the stented valves. The first studies [3-5] on the 3F Enable sutureless aortic valve showed that these prostheses are feasible, safe with an excellent haemodynamic performance. However, the rate of perivalvular leakage remains an important issue with this technology [2]. The first clinical results with the $3 \mathrm{~F}$ Enable sutureless valves were reported in 2008 by Wendt et al. [4]. In their experience, three out of six patients presented perivalvular leakage at follow-up, and one of them underwent successful reoperation with a stented valve after eight months. However, in literature, there are no cases described of sutureless dislocation requiring reintervention. In our experience, the $3 \mathrm{~F}$ Enable was easily removed and also correctly reallocated three months post implantation. The CPB and ACC time were longer than expected. This might certainly be related to our learning curve.

In our opinion, perivalvular leakage, valve dislocation and potential reduction in the CPB and ACC time seem possible with increasing experience and evolution of this technology. The next generation should incorporate more comfortable positioning and quicker deployment.

Further studies are necessary and the durability of this valve has to be investigated in the long-term follow-up.

Conflict of interest: none declared.

\section{REFERENCES}

[1] Bonow RO, Carabello BA, Kanu C, de Leon AC Jr, Faxon DP, Freed MD et al. Guidelines for the Management of Patients with Valvular Heart Disease: a report of the American College of Cardiology/American Heart Association Task Force on Practice Guidelines (writing committee to revise the 1998 Guidelines for the Management of Patients With Valvular Heart Disease): developed in collaboration with the Society of Cardiovascular Anesthesiologists: endorsed by the Society for Cardiovascular Angiography and Interventions and the Society of Thoracic Surgeons. Circulation 2006;114:E84-231.

[2] Martens S, Ploss A, Sirat S, Miskovic A, Moritz A, Doss M. Sutureless aortic valve replacement with the $3 f$ Enable aortic bioprosthesis. Ann Thorac Surg 2009;87:1914-8.

[3] Leyh R, Yildirim C, Buck T, Sommer S, Herold U, Jakob H. Early singlecenter experience with the 3F-Enable aortic valve bioprosthesis. Herz 2006;31:423-8 (German).

[4] Wendt D, Thielmann W, Buck T, Jánosi RA, Bossert T, Pizanis N et al. First clinical experience and 1-year follow-up with the sutureless 3F-Enable aortic valve prosthesis. Eur J Cardiothorac Surg 2008;33:542-7.

[5] Walther T, Simon P, Dewey T, Wimmer-Greinecker G, Falk V, Kasimir MT et al. Transapical minimally invasive aortic valve implantation: multicenter experience. Circulation 2007;116(Suppl 11):I240-5. 